\title{
Einige Briefe von Georg Jacob (1862-1937) an Willi Bang Kaup (1869-1934)
}

\author{
Aloïs van Tongerloo und Michael Knüppel \\ Geel/Kassel
}

\begin{abstract}
In the article the authors give an introduced and commented edition of five letters written by the Turcologist Georg Jacob (1862-1937) to his colleague the Turcologist, Altaist, Iranist and Anglicist Willi Bang Kaup (1869-1934) in the years 1899-1912. In some of the letters Jacob deals with his own works (especially his Turkic dialectological studies and the publication of his works on Karagöz-shadow-play) in some others on the works and scientific activities of another colleague: Karl Foy (1856-1907).
\end{abstract}

Keywords: Georg Jacob, Willi Bang Kaup, correspondencies, edition of letters, history of oriental studies.

Als der Mitbegründer der Turkologie, der Anglist, Iranist und Altaist Willi Bang Kaup (1869-1934) 1914 im Zuge des Ausbruchs des Ersten Weltkrieges Belgien, wo er an der Katholischen Universität Louvain lehrte, verlassen mußte, ließ er aus nicht geklärten Gründen nahezu seine gesamten privaten Korrespondenzen zurück. Diese Materialien haben später, nachdem sie von H. de Vocht (1878-1962), 
einem Schüler und Freund Bangs, geordnet wurden, ${ }^{1}$ als „Nachlaß Bang" ihren Weg in das Archiv der Katholieke Universiteit Leuven gefunden. Dort werden sie heute unter der Signatur PU/ Bang geführt. Die Materialien, die dieser Nachlaß umfaßt, sind für die Geschichte der orientalistischen Sprachwissenschaften ganz allgemein ebenso wie für jene der Orientalistik an der Katholischen Universität Louvain von großem Interesse, erlauben sie doch Einblick in den Wissenschaftsbetrieb des ausgehenden 19. und frühen 20. Jahrhunderts - stand Bang in dieser Zeit doch in Kontakt mit den meisten Vertretern der orientalistischen Disziplinen. Mit einigen bestand dabei eine nur lose Verbindung, mit anderen hingegen ein eher enger Kontakt, von manchen Beziehungen zeugen nur vereinzelte Schreiben, von anderen wiederum Briefwechsel die sich über Jahrzehnte hinzogen und sich in Korrespondenzresten niederschlugen, welche bis zu einhundert Briefe eines einzelnen Korrespondenzpartners, die bei Bang eingingen, umfassen. ${ }^{2}$ Unter den Briefen im Nachlaß Bang finden sich auch fünf Schreiben (oder genauer: vier Briefe und eine Postkarte) eines anderen Mitbegründers der modernen Turkologie: Georg Jacob. ${ }^{3}$ Diese Dokumente werden heute im Nachlaß unter den Nrr. 707-711 (PU/Bang [früher P 56], Karton 39) geführt und stellen den Rest eines einstmals umfangreicheren Briefwechsels zwischen den beiden Gelehrten dar. Die Materialien, die uns einen flüchtigen Blick in die Beziehungen der Korrespondenzpartner erlauben, sollen nachstehend bekannt gemacht resp. erstmals publiziert werden.

Während zu Leben und Werk W. Bang-Kaups inzwischen zahllose Beiträge vorliegen ${ }^{4}$ und an dieser Stelle auf die umfangreicheren biographischen und bio-bibliographischen Arbeiten

\footnotetext{
${ }^{1}$ De Vocht war auch der Herausgeber des Schriftenverzeichnisses von W. Bang Kaup (De Vocht [1929]).

${ }^{2} \mathrm{Zu}$ den Resten dieser sehr umfangreichen Briefwechsel sind die Briefe des Turkologen, Archäologen und Ethnologe A. v. Le Coq (1860-1930), des Iranisten F. C. Andreas (1846-1930) und des Altorientalisten F. H. Weissbach (1865-1944) zu zählen. Die Korrespondenz mit Andreas wurde inzwischen von den Vff.n dieser Zeilen publiziert (Knüppel/van Tongerloo [2012]), eine Edition der Briefe A. v. Le Coqs an W. Bang Kaup wird gerade für die Publikation aufbereitet.

${ }^{3}$ E. Littmann bezeichnete Jacob sogar als „eigentlichen Begründer der Turkologie in Deutschland“" (Littmann [1937], p. 492).

${ }^{4}$ Cf. hierzu die Zusammenstellung in Knüppel/van Tongerloo (2012), bes. den Nachruf von A. v. Gabain (1934) sowie das Schriftenverzeichnis von de Vocht (1929).
} 
verwiesen werden kann, wurde G. Jacob vergleichsweise weniger Aufmerksamkeit zuteil, ${ }^{5}$ sodaß hier zumindest die Eckdaten zu seiner Vita gegeben werden sollen: Georg Jacob wurde am 26.5.1862 im preußischen Königsberg geboren. Er studierte zunächst Theologie und orientalische Sprachen, schließlich Orientalistik, Germanistik und Ethnologie in Leipzig, Straßburg, Breslau und Berlin. 1887 wurde Jacob mit seiner Arbeit „Der nordisch-baltische Handel der Araber im Mittelalter" an der Universität Leipzig promoviert. Die Arbeit, der bereits ein Aufsatz zum selben Gegenstand vorausgegangen war, ${ }^{6}$ erschien im gleichen Jahr. Vom 2.1.1888-1.7.1888 arbeitete Jacob als wissenschaftlicher Hilfsarbeiter, seit dem 31.12.1890 als Assistent an der Königlichen Bibliothek zu Berlin. Mit Wirkung vom 12.2.1892 habilitierte er sich in Greifswald und am 14.11.1896 in Halle a.S. Am 20.2.1901 wurde er zum außerordentlichen Professor in Erlangen und am 7.8.1911 (ab 1.10.1911) zum ordentlichen Professor der Islamischen und Semitischen Philologie, wo er den Lehrstuhl von Georg Hoffmann (1845-1933) übernahm, berufen. Zudem wirkte er von dieser Zeit an als Direktor des Orientalischen Seminars in Kiel. Am 17.12.1916 wurde Jacob zum Geheimen Regierungsrat ernannt und Ende September 1929 entpflichtet. Er starb am 4.7.1937.

Die fünf an Bang gerichteten Schreiben sind insofern von Interesse, als hier eine sehr persönlich eingefärbte Sicht Jacobs auf einen anderen Orientalisten jener Tage, Karl Foy (1856-1907), zur Sprache kommt, und auf diese offenbar eine Reaktion Bangs, die leider nicht mehr vorliegt, erfolgte. Der Vorgang gibt geradezu ein Paradebeispiel für die ,akademischen Befindlichkeiten”, wie sie einem ja auch unserer Tage noch begegnen, ab: Jacob selbst war von den „Verfehlungen” Foys nicht betroffen, und hat unzusammenhängende Vorgänge, von denen er lediglich aus zweiter Hand erfahren hat, zu einer recht unvorteilhaften Charakterisierung des inzwischen verstorbenen Kollegen ausgerechnet einem persönlichen Freund desselben mitgeteilt - ein interessanter Fauxpas und ein aufschlußreiches Lehrstück zugleich!

\footnotetext{
5 Becker (1932); v. Brockdorff (1937); Dammann (1987); Degener (1935); Kürschner's deutscher Gelehrten-Kalender. Jg. 5 1935, p. 377; Kukula (1928); Littmann (1937); Paret/ Schall (1955); Volbehr/ Weyl (1956).

${ }^{6} \mathrm{Jacob}(1886)$.
} 
Dokument 1 (PU/Bang $\mathrm{N}^{\mathrm{r}}$ 707)

Beschreibung

1 Blatt $=1 \mathrm{p}$.

Gesamtmaß des Blattes: $11,2 \mathrm{~cm}$ (br) x $18 \mathrm{~cm}(\mathrm{~h})$.

Seite 1 recto: 18 Z.n (incl. Datum + Unterschrift).

Seite 1 verso: unbeschrieben.

[Seite 1 recto]

Halle ${ }^{\mathrm{a}} / \mathrm{S} 12 / 599$

Hochverehrter Herr Professor,

Besten Dank für Ihre beiden Karten.

Ich beabsichtige mich noch längere Zeit auf das osmanische Türkisch $\mathrm{zu}$ concentriren. In den letzten Monaten habe ich einzig an einem $\mathrm{F}$ vulgärtürkischen Glossar gesammelt. In 8 Tagen etwa hoffe ich Ihnen Heft II meiner Karagöz-Komödien ${ }^{7}$ senden zu können, das einen viel interessanteren Text mit verschiedenen Dialektproben enthält, einige Versehen, welche mir in Heft $\mathrm{I}^{8}$ noch passirt sind, habe ich daselbst berichtigt. Heft III $^{9}$ denke ich mit beigefügter Übersetzung zu geben. Ich würde gerne jetzt Heft auf Heft folgen lassen -6 Stücke habe ich in deutscher Übersetzung im Schreibtisch liegen - weiss aber nicht ob mein Verleger noch weiter geneigt sein wird die Herstellungskosten zu tragen.

Hochachtungsvoll

Ihr ergebenster

Georg Jacob

\footnotetext{
${ }^{7}$ Jacob (1899a).

${ }^{8}$ Jacob (1899b).

${ }^{9}$ Jacob (1899c).
} 
Dokument 2 (PU/Bang $\mathrm{N}^{\mathrm{r}} 708$ )

Beschreibung

1 Blatt $=2$ pp.

Gesamtmaß des Blattes: 11,2 cm (br) x $18 \mathrm{~cm}(\mathrm{~h})$.

Seite 1 recto: 16 Z.n (incl. Datum).

Seite 1 verso: 13 Z.n (incl. Adresse + Unterschrift).

[Seite 1 recto]

Hochverehrter Herr Professor,

Schuchardt's Slawo-Deutsches ${ }^{10}$ besitzt die Bibliothek der DMG nicht, ich benutzte das Exemplar unserer Universitäts-Bibliothek; dieselbe würde Ihnen dasselbe wol auf direkte Eingabe senden, doch dürfte die Sendung eingeschrieben mit Verpackungskosten hin und zurück vielleicht nicht viel billiger werden als das Buch. Unsere türkische Litteratur ist in letzter Zeit durch stetige kleine Ankäufe sehr vermehrt, früher war der Bestand nach dieser Richtung recht dürftig. Der Katalog wird in 14 Tagen erscheinen und trotz seiner 45 Bogen für Mitglieder billig (6-10 Mark) zu haben sein.

Wenn Sie die Güte haben wollten mein Karagözbuch in einer Zeitschrift zu besprechen, ${ }^{11}$ so würde mir das sehr angenehm sein. Wir haben so wenig Turkologen in Deutschland, dass die Besprechungsfrage, von welches doch das Schicksal eines Buches zum grossen Teile abhängt, sehr schwierig ist. Meine Verlags-Buchhandlung ist türkischen Unternehmungen gegenüber sehr ängstlich und so lange sie keinen kleinen Absatz-Erfolg

\footnotetext{
${ }^{10}$ Schuchardt (1884).

${ }^{11}$ Bang scheint den Beitrag zu den Karagöz-Komödien G. Jacob's nicht besprochen zu haben. Er hatte lediglich dessen Beitrag „Zur Grammatik des Vulgär-Türkischen” (Georg [1898]) besprochen - eine Rezension, die zu diesem Zeitpunkt aber wohl schon erschienen war (Bang [1899]).
} 
[Seite 1 verso]

sieht, wird sie mir nicht das 2. Heft kostenfrei drucken, jedes Honorar ist selbstverständlich ausgeschlossen. Das 2. Heft soll die Meddâḥ's behandeln; ich habe einige sehr charakteristische Proben aufgetrieben; neue Aufzeichnungen sind mir aus Stambul versprochen. Die Vermehrung des Materials wäre allerdings wünschenswert; immerhin lässt sich schon sehr viel mehr über diese Stoffe mit Sicherheit sagen als über das Orta ojunu.

Unsere Arabisten sind teilweise entrüstet, dass ich Türkisch treibe! Daher das Vorwort.

Mit hochachtungsvollem Gruss

Ihr ergebenster

Georg Jacob

Halle ${ }^{a} / S$. Kronprinzenstr. 99

Dokument 3 (PU/Bang $\mathrm{N}^{\mathrm{r}}$ 709)

Beschreibung

1 Blatt (gefaltet) $=4$ pp.

Gesamtmaß des Blattes: 22,7 cm (br) x $18 \mathrm{~cm}(\mathrm{~h})$.

Seite 1 recto: 15 Z.n (incl. Datum).

Seite 1 verso: 19 Z.n.

Seite 2 recto: 21 Z.n.

Seite 2 verso: 22 Z.n (incl. Unterschrift).

[Seite 1 recto]

Erlangen 22/9 1909

Verehrter Herr College,

Ihre mir neulich freundlichst übersandte Arbeit enthält S. 236 einen Passus, zu dem ich unmöglich schweigen kann ohne mich einer 
Unterlassung anzuklagen. Sie scheinen über Foys ${ }^{12}$ Persönlichkeit und Lebenswandel nicht genauer unterrichtet zu sein, wenn Sie diesen Mann in solcher Weise zu verherrlichen für angezeigt halten. Ich bin durch Schüler, die aus Berlin kamen und andere über Manches informiert, das mir unmöglich macht mit Hochachtung von diesem Manne zu sprechen. Zur Charakteristik will ich folgende Perfidie mitteilen, die ich selbst erfahren habe. Mein Freund Enno Littmann ${ }^{13}$ fragte mich einst, als er zu einer Orientreise aufbrach, ob er nicht etwas für mich besorgen könne; ich gab ihm ein Blatt mit, auf dem ich einen azer-beiğanischen Text transscribiert und übersetzt hatte und bat ihn bei gelegentlichem Zusammentreffen mit einem Azerbeiğaner die Umschreibung nachzuprüfen. Littmann ging nach dem Orient und ohne dass ich

\section{[Seite 1 verso]}

ihn inzwischen gesehen hatte, nach Amerika, sandte mir von dort den Text mit der Bemerkung, dass er zwar nicht unterwegs, aber in Berlin Gelegenheit gehabt hätte einen Azerbeiğaner zu sprechen. Ich erfuhr durch meinen damals in Berlin weilenden Schüler Philipp, dass ein azerbeiğanischer Buchhändler Hasan, ${ }^{14}$ den ich vor vielen Jahren in Konstantinopel kennengelernt hatte, in Berlin sei und vermutete, dass er dieser war, den Littmann gesprochen*, da Littmann und Philipp in Berlin zusammengekommen waren. Die Transscription liess ich natürlich unter Littmanns Namen später drucken. Etwa ein Jahr darauf wurde Hasan Lector am Orientalischen Seminar. Foy entstellte nun den Sachverhalt in der Weise, dass ich mich an den Leiter des Orient \{alischen\} Seminars gewandt hätte, sodass er Littmanns Namen und die Daten verheimlichte. Wer die Verhältnisse kennt, weiss, dass das eine Niederträchtigkeit war. Wir standen damals noch im Schriftenaustausch. Foy hielt mir diesen Separatabzug vor, obwohl ich

\footnotetext{
${ }^{12}$ Gemeint ist der Orientalist Karl Arthur Philipp Heinrich Foy (1856-1907). Zu Foy cf. Hartmann (1907).

${ }^{13}$ Hier der Semitist, Äthiopist und Turkologe Ludwig Richard Enno Littmann (16.9.1875-4.5.1958). Zu Littmann cf. bes. Biesterfeld (1986).

${ }^{14}$ Es könnte sich hierbei um Ḥasan Taufĩq (1862-1904) handeln, der von 1887-1893 als Lektor für Ägyptisches Arabisch am Seminar für Orientalische Sprachen in Berlin tätig war.
} 
von dem Vorfall hörte und ihn zweimal in Abstand von einigen Monaten um

*) Littmann bestreitet noch heute irrtümlich, dass sein Gewährsmann mit dem Lektor gewordenen Buchhändler identisch sei.

[Seite 2 recto]

denselben bat. Ich bekam inzwischen zuckersüsse schuldbewusste Briefe von ihm. Schliesslich liess ich mir das Elaborat aus München kommen, legte ihm nun ruhig den Sachverhalt dar und verlangte Richtigstellung, obwohl ein unbeabsichtigter Irrtum, da ich Littmann fortwährend zitierte, ausgeschlossen war. Foy hat noch länger als ein halbes Jahr gelebt. Die Berichtigung ist nicht erfolgt!

Die Motive dieser und andere Handlungen kenne ich sehr genau*). Mich hat er vor anständigen Menschen nicht geschädigt. Ich habe deshalb keinen Grund ihm zu grollen, wohl aber ihn zu verachten. Die Tugend von Streberseelen braucht wirklich nicht der Verherrlichung! Gutmütig mag es gewesen sein; gutmütig ist Mancher, der sich die schlimmsten Dinge zu Schulden kommen lässt; das beweist also nichts. Sprachtalent und Scharfsinn mag er besessen haben. Aber seine Arbeiten tragen doch unverkennbar den Stempel völliger Zerrüttung. Eine Wissenschaft, in der man in solchen Zustand noch Grosses leisten konnte, müsste ein trauriges Afterprodukt sein!

*) Auch in anderen Fällen war sich Foy seiner Schuld wohl bewusst, konnte sogar nicht, wie mir von Zeugen berichtet wurde, die äussere Verwirrung und Scham verbergen, wenn man ihn danach fragte.

[Seite 2 verso]

Die türkische Geschichte trug Foy in der Weise vor, dass es von den ersten osmanischen Herrschern auf die Reformen Mahmud II überging, ${ }^{15}$ natürlich nach den trübsten Quellen, die vielleicht in letzter Instanz auf $\{\mathrm{J}$. von $\}$ Hammer $\left\{-\right.$ Purgstall ${ }^{16}$ zurückgingen,

\footnotetext{
${ }^{15}$ Gemeint ist der osman. Sulțān-Halīf Mahmūd II. (1808-1839).

${ }^{16}$ Hier der Begründer der Osmanistik und Mitbegründer der Orientalistik Joseph Freiherr v. Hammer-Purgstall (9.6.1774-23.11.1856).
} 
dabei meist in jede Stunde wieder mit demselben anfangend, da sein Gedächnis nicht mehr bis zur letzten reichte. Sein „Anonymus“ hat eine so willkürlich=freie Umschrift, dass es dieselben Worte, wenn sie zwei- oder dreimal vorkommen, stets so verschieden wie möglich wiedergiebt. Ein ,vortrefflicher Philologe' baut auf solches Material keine Kartenhäuser. Bei einer so krassen Unkenntnis der Sachen,*) wie sie Foy besass, ist es mislich mit Lauten zu operieren. Der „Anonymus" erwähnt die Hurûfîs als horife, wenn ich nicht irre; von Hurûfîs wusste natürlich Foy nichts, er denkt an ${ }^{\mathrm{c}}$ ārif, und ein famoses Lautgesetz $\mathrm{h}={ }^{\mathrm{c}}$ ist fertig. Foy hatte zudem am Seminar nicht ein $[\ldots]^{17}$ Prähistorische umgedrehtes türkisches Volapük, von ihm erfunden, zu vertreten, sondern osmanisches Türkisch. Da hätte er sich wohl etwas mit den Elementen des Arabischen auseinandersetzen müssen, in denen er so unsicher war, dass er Studenten bei den bekanntesten Worten [...]lich ${ }^{18}$ fragen musste, ob sie mit oder zu schreiben seien.
In vorzüglicher Hochachtung
Ihr erg $\{$ ebener $\}$
Georg Jacob

*) In seiner Compassarbeit kennt er die ganze alte Litteratur nicht, die Alles von ihm gesagte gegenstandslos macht.

\section{Dokument 4 (PU/Bang N ${ }^{\mathrm{r}}$ 710)}

Beschreibung

1 Blatt (gefaltet) $=2 \mathrm{pp}$.

Gesamtmaß des Blattes: 22,7 cm (br) x 18 cm (h).

Seite 1 recto: 18 Z.n (incl. Datum).

Seite 1 verso: 4 Z.n (incl. Unterschrift).

Seite 2 recto: unbeschrieben.

Seite 2 verso: unbeschrieben.

\footnotetext{
${ }^{17}$ Lesung unklar.

${ }^{18}$ Lesung unklar.
} 
[Seite 1 recto]

Erlangen 25/9 1909

Verehrter Herr College,

Es tut mir leid, dass Ihnen mein Brief weh getan hat. Ich wusste weder, dass Sie sich Foy gegenüber zu Dank verpflichtet fühlen, noch dass Sie ihn, wie es scheint, garnicht persönlich kannten. Ihre Auslassung kam mir herausfordernd vor, und riss bei mir alte Wunden auf. Ich will mich jetzt bemühen jede gereizte Stimmung zu unterdrücken.

Littmann mochte Foy gern; ich habe ihm daher um mich vor Übereilung zu hüten vor dem definitiven Bruch, als er mich hier einmal besuchte, die ganze Correspondenz ohne weiteren Kommentar vorgelegt; er sagte nach Kenntnisnahme: „Ich habe Foy das nicht zugetraut." Foy kam immer mit Entschuldigungen, ich wollte eine Richtigstellung; er hat mir dieselbe nie versprochen, also habe ich keinen Grund anzunehmen, dass eine solche verloren gegangen ist. Das mitgeteilte Beispiel seiner incorrecten Handlungsweise mir gegenüber war allerdings das, was für mich durch die Umstände am meisten ins Gewicht fiel, aber es steht nicht isoliert.

Sonst kann ich dem, was Sie schreiben, nur zustimmen und ich bitte nochmals um Entschuldigung, wenn ich Sie irgendwie verletzt haben sollte, was

[Seite 1 verso]

nicht meine Absicht war.

In aufrichtiger Hochachtung

Ihr

Georg Jacob 
Dokument 5 (PU/Bang $\mathrm{N}^{\mathrm{r}}$ 711)

Postkarte

Gesamtmaß der Postkarte: 14 cm (br) x 9,1 cm (h).

verso: 10 Z.n (incl. Datum + Unterschrift).

[Recto Seite]

/Poststempel:/

KIEL 8.7.12

/Anschrift:/

Herrn Professor Bang, Loewen, Rue des Récollets.

[Verso Seite]

Geschrieben von WBK: $\quad\{$ W. $\} \operatorname{Radl}\{$ off $\}$ sin, syn

Kiel 8/7 1912

Joh \{ann $\}$ Georgstr\{asse $\} 13^{\text {II }}$

Verehrter Herr College, at

Leider habe ich hier keine Hülfsmittel für türkische Dialekte und besitze selbst fast nur Osmanica. Evlija berichtet, dass man in Adrianopel für Kirchhof sinbaz sage; ich kenne sin nur als veraltetes Wort für Grab, baz sagen die Tataren für čukur (Grube); ist Ihnen die Zusammensetzung sonst bekannt. Für lachen sagte man in Adrianopel ak- atlanmak,

/in margine HS WBK:/ aq + at 
für sich amüsieren kyscharlanmak. Wir haben wunderbare Sachen geschaut hier: alata serejân ettik. Steckt darin vielleicht auch altes Material?

Mit bestem Gruss $\quad$ Ihr er \{gebener $\}$

Georg Jacob

\section{Literatur}

Bang, Willi (1899): [Bespr. v.]: G. Jacob: Zur Grammatik des VulgärTürkischen. In: WZKM XIII, pp. 110-113.

Becker, C. H.: Georg Jacob als Orientalist. In: Menzel, Theodor (Hrsg.): Festschrift Georg Jacob zum siebzigsten Geburtstag 26. Mai 1932 gewidmet von Freunden und Schülern. Leipzig 1932, pp. 369-381.

Biesterfeld, Hans Hinrich: Enno Littmann: Leben und Arbeit. Ein autobiographisches Fragment (1875-1904). In: Oriens 29. 1986, pp. $1-101$.

Brockdorff, Cay v. (1937): 1. Wahrheit und Wahrscheinlichkeit bei Hobbes und Condillac. 2. Inward veracity in its religious sense. Kiel (Veröffentlichungen der Hobbes-Gesellschaft 8), pp. 28 31.

Dammann, Ernst (1987): Erinnerungen an Georg Jacob. In: Klaus Kreiser (Hrsg.): Germano-Turcica. Zur Geschichte des Türkischlernens in den deutschsprachigen Ländern: Ausstellung des Lehrstuhls für Türkische Sprache, Geschichte und Kultur der Universität Bamberg in Zusammenarbeit mit der Universitätsbibliothek Bamberg: Universitätsbibliothek, 7.31.7. 1987. Bamberg 1987, pp. 113-118.

Degener, Hermann A. L. (1935): Degeners Wer ist's? Eine Sammlung von rund 18.000 Biographien mit Angaben über Herkunft, Familie, Lebenslauf, Veröffentlichungen und Werke, Lieblingsbeschäftigung, Mitgliedschaft bei Gesellschaften, Anschrift und anderen Mitteilungen von allgemeinem Interesse. 10. Ausg., p. 345. 
Gabain, Annemarie von (1934): W. Bang Kaup $†$. In: UAJb 14, pp. 335-340.

Hartmann, Martin (1907): Karl Foy, 1856-1907. In: MSOSW 10, pp. 299-304.

Jacob, Georg (1886): Welche Handelsartikel bezogen die Araber des Mittelalters aus den nordisch-baltischen Ländern? Berlin [2. Aufl. Berlin 1891].

Jacob, Georg (1887): Der nordisch-baltische Handel der Araber im Mittelalter. Leipzig.

Jacob, Georg (1898): Zur Grammatik des Vulgär-Türkischen. In: ZDMG 52, pp. 695-729.

Jacob, Georg (1899a): Kajyk ojunu. Türkischer Text mit Anmerkungen und einer Einleitung versehen. Berlin (Karagöz-Komödien 2).

Jacob, Georg (1899b): Schejtan dolaby. Türkischer Text mit Anmerkungen herausgegeben und mit einer Einleitung über das islamische Schattenspiel versehen. Berlin (Karagöz-Komödien 1).

Jacob, Georg (1899c): Die Akserai-Schule. Fünf Karagöz-Stücke. Berlin (Karagöz-Komödien 3).

Knüppel, Michael/van Tongerloo, Aloïs (2012): Die orientalistische Gelehrtenrepublik am Vorabend des Ersten Weltkrieges. Der Briefwechsel zwischen Willi Bang(-Kaup) und Friedrich Carl Andreas aus den Jahren 1889 bis 1914. Berlin (Abhandlungen der Akademie der Wissenschaften zu Göttingen N.F. 20).

Kürschner's deutscher Gelehrten-Kalender. Jg. 5 1935, p. 377.

Kukula, Richard (1928): Wer ist's? 9. 1928.

Littmann, Enno (1937): Georg Jacob† (1862-1937). In: ZDMG 91, pp. 496-500.

Paret, Rudi/Schall, Anton (1955): Ein Jahrhundert Orientalistik. Lebensbilder aus der Feder von Enno Littmann und Verzeichnis seiner Schriften. Zum 80. Geburtstage am 16.IX.1955, pp. 96109. 
Schuchardt, Hugo (1884): Dem Herrn Franz von Miklosich zum 20. November 1883. Slawo-Deutsches und Slawo-Italienisches. Graz.

De Vocht, Henri (1929): Bibliographie der Arbeiten von Professor W. Bang Kaup. In: UJb IX, pp. 188-195.

Volbehr, Friedrich/Weyl, Richard (1956): Professoren und Dozenten der Christian-Albrechts-Universität zu Kiel 1665-1954. Mit Angaben über die sonstigen Lehrkräfte und die Universitätsbibliothekare und einem Verzeichnis der Rektoren. 4. Aufl. Kiel, p. 158. 